Gut and Liver, Vol. 11, No. 4, July 2017, pp. 451-452

\title{
Does Single Balloon Enteroscopy Have Similar Efficacy and Endoscopic Performance Compared with Double Balloon Enteroscopy?
}

\author{
Hyun Joo Jang \\ Department of Internal Medicine, Hallym University School of Medicine, Hwasung, Korea
}

See "Comparison of the Efficacy and Safety of Single- versus Double-Balloon Enteroscopy Performed by Endoscopist Experts in Single-Balloon Enteroscopy: A Single-Center Experience and Meta-Analysis" by Tae Jun Kim, et al. on page 520, Vol. 11. No. 4,2017

The small bowel had been considered unobservable area for gastrointestinal endoscopy, as most of the small bowel was inaccessible with conventional endoscopy. The advent of capsule endoscopy and balloon-assisted enteroscopy during the last decade revolutionized the diagnosis and management of small bowel diseases. Capsule endoscopy, developed in 2001, has been evolved as the main technique for the endoscopic evaluation of small bowel disorders because it is a noninvasive and safe technique for complete small bowel visualization. However, it has several major limitations, lack of therapeutic capabilities and movement control.

Yamamoto et al. ${ }^{1}$ described the double balloon enteroscopy (DBE) technique visualizing small intestine and capable of therapeutic procedures in 2001. DBE has one latex balloon to the tip of enteroscope and the other on the tip of overtube. The balloon of the tip of enteroscope and one of the overtube are alternatively inflated or deflated with air from a pressure-controlled pump unit of the DBE system. The mechanism of DBE was based on the concept that stretching of the small bowel during the insertion of endoscope prevents further endoscopic advancement, and that the usage of two balloons of enteroscope tip and overtube would hold the intestinal wall and prevent subsequent loop formation and make further advancement of enteroscopy. ${ }^{1}$

In 2007, single balloon enteroscopy (SBE) technique was developed, in which a latex-free balloon is attached to the tip of the silicon overtube and is inflated or deflated with air controlled by a balloon control system. ${ }^{2}$ Preparation and operation of DBE are more time-consuming and cumbersome, manually applying a latex balloon to enteroscope tip as well as inflation and deflation of two balloons than one of SBE. SBE may provide a quicker procedure with shorter procedure time. ${ }^{3}$ However SBE may have some difficulties to perform deep insertion of enteroscopy due to the absence of a balloon of enteroscope tip to prevent small bowel getting free. A randomized controlled trial reported that total enteroscopy rate of DBE is higher than one of SBE. ${ }^{4}$

Despite of lower rate of total enteroscopy, recent studies demonstrated that SBE and DBE have similar diagnostic, therapeutic yields and insertion depth in the patients with suspected small bowel diseases. ${ }^{5,6}$ There are two meta-analyses about diagnostic and therapeutic yields, and endoscopic performance of DBE and SBE, which also reported that both DBE and SBE have similar diagnostic and therapeutic yields.,

In this issue of Gut and Liver, Kim et al. ${ }^{7}$ reported the retrospective analysis of SBE and DBE data by a single enteroscopist of a single center and meta-analysis of the efficacy and safety of SBE and DBE. From a prospective balloon-assisted enteroscopy registry, a total of 65 enteroscopic procedures in 44 patients with SBE and 73 procedures in 69 patients with DBE were included. The results of this issue reported that there were no significant differences in diagnostic yields, therapeutic yields and complication rate between DBE and SBE in their enteroscopy registry. ${ }^{7}$ Kim et al. also analyzed the meta-analysis including four small-scale randomized-controlled trials and three observational studies. This meta-analysis also demonstrated that there were no significant differences in the pooled relative risk and odds ratio for diagnostic and therapeutic yield and complication between SBE and DBE. ${ }^{7}$ Previous meta-analyses including four

Correspondence to: Hyun Joo Jang

Division of Gastroenterology and Hepatology, Department of Internal Medicine, Hallym University School of Medicine, 7 Keunjaebong-gil, Hwaseong 18450, Korea

Tel: +82-31-8086-2450, Fax: +82-31-8086-2029, E-mail: jhj1229@hallym.or.kr

pISSN 1976-2283 eISSN 2005-1212 https://doi.org/10.5009/gnl17225

@ This is an Open Access article distributed under the terms of the Creative Commons Attribution Non-Commercial License (http://creativecommons.org/licenses/by-nc/4.0) which permits unrestricted non-commercial use, distribution, and reproduction in any medium, provided the original work is properly cited. 
randomized controlled studies reported similar results to this issue. $^{5,6}$ Meta-analysis of this issue included three large-scale of observations studies as well as four randomized controlled studies. The author suggested the large-scale of observational studies might reflect real practice of DBE and SBE. ${ }^{7}$

There are some limitations of this issue. This study is a retrospective study of comparison between SBE and DBE which might have some bias. The results might be dependent on which one was that the endoscopists had the experiences to overcome learning curve at the first time if the endoscopists do not have the same volume of experiences on DBE and SBE. Although there are some limitations, it seems that SBE shows similar diagnostic, therapeutic yields and complication rate to DBE. Either DBE or SBE can be used for the diagnosis or treatment of small intestinal diseases depending on the available endoscopic system in the hospital on the basis of this result. Well-designed randomized controlled trials of large sample size to compare these techniques are still needed to demonstrate the efficacy of both enteroscopy.

\section{CONFLICTS OF INTEREST}

No potential conflict of interest relevant to this article was reported.

\section{REFERENCES}

1. Yamamoto H, Sekine Y, Sato Y, et al. Total enteroscopy with a nonsurgical steerable double-balloon method. Gastrointest Endosc 2001;53:216-220

2. Kawamura T, Yasuda K, Tanaka K, et al. Clinical evaluation of a newly developed single-balloon enteroscope. Gastrointest Endosc 2008;68:1112-1116.

3. Lenz P, Roggel M, Domagk D. Double- vs. single-balloon enteroscopy: single center experience with emphasis on procedural performance. Int J Colorectal Dis 2013;28:1239-1246.

4. Takano N, Yamada A, Watabe H, et al. Single-balloon versus double-balloon endoscopy for achieving total enteroscopy: a randomized, controlled trial. Gastrointest Endosc 2011;73:734-739.

5. Wadhwa V, Sethi S, Tewani S, et al. A meta-analysis on efficacy and safety: single-balloon vs. double-balloon enteroscopy. Gastroenterol Rep (Oxf) 2015;3:148-155.

6. Lipka S, Rabbanifard R, Kumar A, Brady P. Single versus double balloon enteroscopy for small bowel diagnostics: a systematic review and meta-analysis. J Clin Gastroenterol 2015;49:177-184.

7. Kim TJ, Kim ER, Chang DK, Kim YH, Hong SN. Comparison of the efficacy and safety of single- versus double-balloon enteroscopy performed by endoscopist experts in single-balloon enteroscopy: a single-center experience and meta-analysis. Gut Liver 2017;11:520-527. 